\title{
Examples of non-rigid CAT(0) groups from the category of knot groups
}

\author{
Christopher Paul MoOney
}

\begin{abstract}
C Croke and B Kleiner have constructed an example of a CAT $(0)$ group with more than one visual boundary. J Wilson has proven that this same group has uncountably many distinct boundaries. In this article we prove that the knot group of any connected sum of two non-trivial torus knots also has uncountably many distinct CAT(0) boundaries.
\end{abstract}

57M07, 20F65

\section{Introduction}

The CAT $(0)$ condition is a geometric notion of nonpositive curvature similar to the definition of Gromov $\delta$-hyperbolicity. A proper geodesic space $X$ is called CAT( 0$)$ if it has the property that geodesic triangles in $X$ are "no fatter" than geodesic triangles in Euclidean space (a precise definition is given by MR Bridson and A Haefliger in [4, Chapter II.1]). The visual or ideal boundary of $X$, denoted $\partial X$, is the collection of endpoints of geodesic rays emanating from a chosen basepoint endowed with the cone topology. It is well-known that $\partial X$ is well-defined and independent of choice of basepoint and that $X \cup \partial X$ is a $Z$-set compactification for $X$. A group $G$ is called CAT $(0)$ if it acts geometrically (ie properly discontinuously and cocompactly by isometries) on some CAT( 0$)$ space $X$. In this setup we call $X$ a CAT(0) $G$-space and $\partial X$ a CAT(0) boundary of $G$. We say that a CAT( 0$)$ group $G$ is rigid if it has only one topologically distinct boundary.

It is well-known that if $G$ is negatively curved (acts geometrically on a Gromov $\delta-$ hyperbolic space) or if $G$ is free abelian then $G$ is rigid. Apart from this little is known concerning rigidity of groups. PL Bowers and K Ruane showed that if $G$ splits as the product of a negatively curved group with a free abelian group then $G$ is rigid [3]. Ruane proved later in [9] that if $G$ splits as a product of two negatively curved groups then $G$ is rigid. T Hosaka has extended this work to show that in fact it suffices to know that $G$ splits as a product of rigid groups [7]. Another condition which guarantees rigidity is knowing that $G$ acts on a $\operatorname{CAT}(0)$ space with isolated flats which was proven by $\mathrm{C}$ Hruska in [8]. 
Not all CAT(0) groups are rigid, however: C Croke and B Kleiner constructed in [5] an example of a non-rigid CAT(0) group $G$. Specifically, they showed that $G$ acts on two different CAT(0) spaces whose boundaries admit no homeomorphism. J Wilson proved in [11] that this same group has uncountably many boundaries.

In this article we exhibit an infinite family of non-rigid knot groups. It is a Corollary of Thurston's hyperbolization theorem [10] for Haken 3-manifolds that every knot is either a torus knot, a hyperbolic knot or a satellite knot. It follows from Hruska's result [8] that hyperbolic knot groups are rigid. Furthermore, using a result of T Bedenikovic, A Delgado and M Timm [2] and the Bowers-Ruane result from [3] we can prove that torus knot groups are rigid (see Proposition 4.5). The following theorem gives us an infinite family of non-rigid satellite knots.

Theorem 1 The knot group $G$ of any connected sum of two non-trivial torus knots has uncountably many $C A T(0)$ boundaries.

Specifically, we will prove that given any such knot group $G$, there is a natural construction of a family of CAT(0) $G$-spaces which is analogous to the construction used by Croke and Kleiner in [5]. Even though each space here will have a similar but significantly different structure from the spaces constructed in [5] (see Section 4.3), we will show that on the level of boundaries they have the same basic properties. Interestingly enough, it turns out that the proof given in [5] will not work in this situation. In order to get any results we will require the work of Wilson [11]. This is discussed in more detail at the end of Section 2.

As a final comment on the statement of Theorem 1, there is a stronger notion of rigidity than the definition we use here. Sometimes a CAT(0) group is said to be rigid if every $G$-equivariant quasi-isometry between two CAT( $(0)$ spaces extends to a homeomorphism of the boundaries. For us such a group will be called strongly rigid. Negatively curved groups are strongly rigid, for instance. The fact that these two notions of rigidity are distinct is due to Bowers and Ruane who exhibit in [3] an example of a group which is rigid (that is, weakly rigid) but not strongly rigid.

In [6] Croke and Kleiner found necessary and sufficient conditions for determining when the fundamental group of a 3-dimensional graph manifold is strongly rigid. Since the groups we are considering fall under this category, our result is slightly stronger than theirs for this particular class of groups. We prove that the knot group of any connected sum of two non-trivial torus knots is not even weakly rigid.

\subsection{Acknowledgements}

The work contained in this paper is published as one part of the author's $\mathrm{PhD}$ thesis written under the direction of Craig Guilbault at the University of Wisconsin-Milwaukee. 
The author would like to thank the referee for their helpful suggestions along with Ric Ancel, Chris Hruska, Boris Okun and Tim Schroeder.

\section{Croke and Kleiner's original construction}

Before diving into the proof of Theorem 1, we quickly sketch the proof of the main theorem of [5]. Let $G=G_{C K}$ be the group given by the presentation:

$$
\langle a, b, c, d \mid a b=b a, b c=c b, c d=d c\rangle .
$$

Croke and Kleiner construct CAT(0) $G$-spaces $X$ such that each $X$ is covered by a collection of closed convex subspaces called blocks. The visual boundary $\partial B$ of every block $B$ is the suspension of a Cantor set. The suspension points are called poles. If two blocks $B_{0}$ and $B_{1}$ intersect, then $B_{0}$ is said to neighbor $B_{1}$ and their intersection is a Euclidean plane called a wall. They then prove five statements for each $X$.

Theorem A [5, Section 1.4] The nerve $N$ of the collection of blocks is a tree.

Theorem B [5, Lemma 3] Let $B_{0}$ and $B_{1}$ be blocks and $D$ be the distance between the corresponding vertices in $N$. Then the following hold.

(1) If $D=1$, then $\partial B_{0} \cap \partial B_{1}=\partial W$ where $W$ is the wall $B_{0} \cap B_{1}$.

(2) If $D=2$, then $\partial B_{0} \cap \partial B_{1}$ is the set of poles of $B_{\frac{1}{2}}$ where $B_{\frac{1}{2}}$ intersects $B_{0}$ and $B_{1}$.

(3) If $D>2$, then $\partial B_{0} \cap \partial B_{1}=\varnothing$.

A local path component of a point in a space is a path component of an open neighborhood of that point.

Theorem C [5, Lemma 4] Let $B$ be a block and $\zeta \in \partial B$ not be a pole of any neighboring block. Then $\zeta$ has a local path component which stays in $\partial B$.

Theorem D [5, Corollary 8] The union of block boundaries in $\partial X$ is the unique dense safe path component of $\partial X$.

The definition of safe path will be given in Section 5.3. For now it suffices to understand that Theorem D gives a way to topologically distinguish the union of block boundaries in $\partial X$. With these theorems in hand it is not hard to prove that given two constructions $X_{1}$ and $X_{2}$, any homeomorphism $\partial X_{1} \rightarrow \partial X_{2}$ takes poles to poles, block boundaries to block boundaries and wall boundaries to wall boundaries. The last piece of the 
puzzle is Theorem E. Given $0<\theta \leq \pi / 2$, we can construct $X_{\theta}$ in such a way that the minimum Tits distance between poles is $\theta$. For a block $B$, we denote by $\Pi B$ the set of poles of neighboring blocks.

Theorem E [5, Lemma 9] (also [11, Proposition 2.2]) For a block $B$, the union of boundaries of walls of $B$ is dense in $\partial B$ and $\overline{\Pi B}$ is precisely the set of points of $\partial B$ which are a Tits distance of $\theta$ from a pole of $B$.

With these five theorems in hand we get the main result of [5].

Theorem CK Let $B$ be a block and $L$ be a suspension $\operatorname{arc}$ of $\partial B$. Then $|L \cap \overline{\Pi B}|=1$ iff $\theta=\pi / 2$. Therefore $G_{C K}$ has at least two distinct boundaries.

In [11] Wilson uses these five theorems to prove a stronger result.

Theorem W If $\theta_{1} \neq \theta_{2}$, then $\partial X_{\theta_{1}} \not \approx \partial X_{\theta_{2}}$. Therefore $G_{C K}$ has uncountably many distinct boundaries.

In this article we consider the knot group $G=G_{K}$ of any connected sum $K$ of torus knots. We produce for $G$ an analogous family of CAT(0) spaces which have a similar structure to those constructed in [5]. Specifically, we have blocks, walls and poles for these spaces as well and for each $0<\theta<\pi / 2$ we can construct $X_{\theta}$ such that the minimum Tits distance between two poles is $\theta$. This done, we show that we have the appropriate analogues to Theorem A-Theorem E.

Now if we had $X_{\pi / 2}$, then Theorem A-Theorem E would be enough to guarantee that $G$ has at least two boundaries. Thus we would not need the arguments found in [11] to prove that $G$ is not rigid. However, as we will see in Proposition 4.4 there is no "natural" construction which will yield $X_{\pi / 2}$. Therefore in order to prove that $G$ is not rigid we really need to apply the work of [11].

\section{Block structures on CAT(0) spaces}

We begin by observing that the work in [5, Sections 1.4-5] does not depend on the specific construction used in in [5]. The same observations apply if we replace their definition of a block with the following one.

Definition 3.1 Let $X$ be a CAT( 0 ) space and $\mathcal{B}$ be a collection of closed convex subspaces covering $X$. We call $\mathcal{B}$ a block structure on $X$ and its elements blocks if $\mathcal{B}$ satisfies the following three properties. 
(1) Every block intersects at least two other blocks.

(2) Every block has a (+) or (-) parity such that two blocks intersect only if they have opposite parity.

(3) There is an $\epsilon>0$ such that two blocks intersect iff their $\epsilon$-neighborhoods intersect.

The nerve of a collection $\mathcal{C}$ of sets is the (abstract) simplicial complex with vertex set $\left\{v_{B} \mid B \in \mathcal{C}\right\}$ such that a simplex $\left\{v_{B_{1}}, \ldots, v_{B_{n}}\right\}$ is included whenever $\bigcap_{i=1}^{n} B_{i} \neq \varnothing$. In exactly the same way as in [5] the nerve $\mathcal{N}$ of the collection of blocks is a tree, and we can define the itinerary of a geodesic. A geodesic $\alpha$ is said to enter a block if it passes through a point which is not in any other block. The itinerary of $\alpha$ is defined to be the list $\left[B_{1}, B_{2}, \ldots\right]$ where $B_{i}$ is the $i$ th block that $\alpha$ enters. This list is denoted by Itin $\alpha$. The following lemma follows in the same way as [5, Lemma 2], which simply uses the fact that a block $B$ is convex and that its topological frontier is covered by the collection of blocks corresponding to the link in $\mathcal{N}$ of the vertex $v_{B}$.

Lemma 3.1 If Itin $\alpha=\left[B_{1}, B_{2}, \ldots\right]$, then $\left[v_{B_{1}}, v_{B_{2}}, \ldots\right]$ is a geodesic in $\mathcal{N}$.

We may also talk about the itinerary between two blocks. If $\left[v_{B_{1}}, \ldots, v_{B_{n}}\right]$ is the geodesic edge path in $\mathcal{N}$ connecting two vertices $v_{B_{0}^{\prime}}$ and $v_{B_{1}^{\prime}}$, then we call $\left[B_{1}, \ldots, B_{n}\right]$ the itinerary between $B_{0}^{\prime}$ and $B_{1}^{\prime}$ and write:

$$
\operatorname{Itin}\left[B_{0}^{\prime}, B_{1}^{\prime}\right]=\left[B_{1}, \ldots, B_{n}\right] .
$$

The two notions of itineraries are related as follows: The itinerary of a geodesic segment $\alpha$ is the shortest itinerary $\operatorname{Itin}\left[B_{0}^{\prime}, B_{1}^{\prime}\right]$ for which $\alpha$ begins in $B_{0}^{\prime}$ and ends in $B_{1}^{\prime}$. Note also that the same observations which gave us Lemma 3.1 also provide this next lemma.

Lemma 3.2 Let $B_{0}^{\prime}$ and $B_{1}^{\prime}$ be blocks, write $\operatorname{Itin}\left[B_{0}^{\prime}, B_{1}^{\prime}\right]=\left[B_{1}, \ldots, B_{n}\right]$, and let $\alpha$ be a geodesic beginning in $B_{0}^{\prime}$ and ending in $B_{1}^{\prime}$. Then the following hold.

(1) $\alpha$ enters $B_{k}$ for every $1<k<n$.

(2) $\alpha$ passes through $B_{k} \cap B_{k+1}$ for every $1 \leq k<n$.

(3) $\bigcup_{k=1}^{n} B_{k}$ is convex.

We call a geodesic ray rational if its itinerary is finite and irrational if its itinerary is infinite. A point of $\partial X$ is called irrational if it is the endpoint of an irrational geodesic ray; otherwise we call it rational. We denote the set of rational points of $\partial X$ by $R X$ and the set of irrational points by $I X$. 
Lemma 3.3 Let $\alpha$ be an irrational geodesic ray. Then for any block $B_{0}$

$$
\lim _{t \rightarrow \infty} d\left(\alpha(t), B_{0}\right)=\infty
$$

Proof Write Itin $\alpha=\left[B_{1}, B_{2}, \ldots\right]$. Since $\mathcal{N}$ is a tree we can find $M>1$ such that for every $m \geq M, \operatorname{Itin}\left[B_{0}, B_{m}\right] \ni B_{M}$. For $m \geq M$ choose a time $t_{m}$ such that $\alpha\left(t_{m}\right) \in B_{m}$. Then:

$$
\begin{aligned}
\lim _{t \rightarrow \infty} d\left(\alpha(t), B_{0}\right) & \geq \lim _{t \rightarrow \infty} d\left(\alpha(t), B_{M}\right) \\
& =\lim _{m \rightarrow \infty} d\left(\alpha\left(t_{m}\right), B_{M}\right) \\
& \geq \lim _{m \rightarrow \infty} d\left(B_{m}, B_{M}\right) .
\end{aligned}
$$

Hence it suffices to prove the following claim.

Claim Let $\epsilon$ be given as in condition (3) of Definition 3.1. Then whenever $d\left(v_{B}, v_{B^{\prime}}\right) \geq 2 k$, we have $d\left(B, B^{\prime}\right) \geq 2 k \epsilon$.

Note that whenever $d\left(v_{B}, v_{B^{\prime}}\right)=2$ then we have $d\left(B, B^{\prime}\right) \geq 2 \epsilon$ because the $\epsilon-$ neighborhoods of $B$ and $B^{\prime}$ do not overlap. Assume $\operatorname{Itin}\left[B, B^{\prime}\right]=\left[B_{0}, B_{1}, \ldots, B_{n}\right]$ where $n \geq 2 k$. Then for any $x \in B$ and $x^{\prime} \in B^{\prime}$ the geodesic $\left[x, x^{\prime}\right]$ passes through $B_{2 i}$ for $0 \leq i \leq k$ at some point $z_{i}$. So

$$
d\left(x, x^{\prime}\right)=\sum_{i=0}^{k-1} d\left(z_{i}, z_{i+1}\right) \geq 2 k \epsilon .
$$

\section{Corollary 3.1}

(1) $R X$ is the union of block boundaries in $\partial X$ and $I X$ is its complement.

(2) If $\zeta \in I X$, then every geodesic ray going out to $\zeta$ is irrational.

(3) If $\zeta \in I X$ and $\alpha$ and $\beta$ are geodesic rays going out to $\zeta$, then the itineraries of $\alpha$ and $\beta$ eventually coincide.

A geodesic space is said to have the geodesic extension property if every geodesic segment can be extended to a geodesic line. As is true with the original Croke-Kleiner construction, the blocks we construct will satisfy the geodesic extension property.

Lemma 3.4 If blocks have the geodesic extension property, then $R X$ is dense.

Proof Let $\alpha$ be an irrational geodesic ray and write Itin $\alpha=\left[B_{1}, B_{2}, \ldots\right]$. For each $n \geq 1$ let $t_{n}$ be a time at which $\alpha\left(t_{n}\right) \in B_{n}$. Then every ray $\left.\alpha\right|_{\left[0, t_{n}\right]}$ can be extended to a geodesic ray $\alpha_{n}$ which does not leave the block $B_{n}$. Then $\alpha_{n} \rightarrow \alpha$. 
We end this section with a definition which will simplify the proof of Theorem $\mathrm{D}^{\prime}$ later. Given a space $Y$ we call a surjective map $\phi: I X \rightarrow Y$ an irrational map if it satisfies the property that $\phi(a)=\phi(b)$ iff whenever $\alpha$ and $\beta$ are geodesic rays going out to $a$ and $b$ respectively then Itin $\alpha$ and Itin $\beta$ eventually coincide. The obvious candidate for such a map is the function $\phi: I X \rightarrow \partial \mathcal{N}$ which takes $a$ to the boundary point in $\partial \mathcal{N}$ determined by the itinerary of a ray going out to $a$. This function is well-defined by Corollary 3.1(3). All we need to know is that $\phi$ is continuous, which amounts to proving the following lemma.

Lemma 3.5 Let $\left(\alpha_{n}\right)$ be a sequence of irrational rays with common basepoint converging to another irrational ray $\alpha$. Then for every $B \in \operatorname{Itin} \alpha$ we have $B \in \operatorname{Itin} \alpha_{n}$ for large enough $n$.

Proof Write Itin $\alpha=\left[B_{1}, B_{2}, \ldots\right]$ and choose $k \geq 1$. Then $B_{k+1}$ is a neighborhood of $\alpha(t)$ for some time $t$, which means that for large enough $n \alpha_{n}(t) \in B_{k+1}$. Since $\left.\alpha_{n}\right|_{[0, t]}$ begins in $B_{1}$ and ends in $B_{k+1}$, Lemma 3.2(1) tells us that it must enter $B_{k}$.

Corollary 3.2 The natural map $\phi: I X \rightarrow \partial \mathcal{N}$ determined by itineraries is an irrational map.

\section{CAT(0) knot groups}

\subsection{Preliminary definitions}

Before we begin discussing knot groups we present some standard terminology concerning CAT( 0$)$ groups. Greater detail is given by Bridson and Haefliger [4, Chapter II.6]. Let $h$ be an isometry of a CAT(0) space $Z$. If there is a geodesic line $L$ such that $h$ restricts to a non-trivial translation of $L$, then $L$ is called an axis of $h$. For a point $z \in L$ the sequence $\left(h^{n} z\right)_{n=1}^{\infty}$ converges in $Z \cup \partial Z$ to one of the two boundary points of $L$; we call that boundary point $h^{\infty}$. In fact, given any $z \in Z$ the sequence $\left(h^{n} z\right)$ converges to the same point $h^{\infty}$.

The minset of an element $g \in G$, written Min $g$, is the subspace of $Z$ where the map $z \mapsto d(z, g z)$ achieves its minimum. If there is an element $h_{0}$ in the center of $G$, then $\operatorname{Min} h_{0}$ is the union of axes of $h_{0}$ and $\operatorname{Min} h_{0}$ splits as a CAT(0) product $Z^{\prime} \times \mathbb{R}$. In this structure, the axes of $h_{0}$ are the geodesic lines $\{z\} \times \mathbb{R}$.

There are two notions of angles in $\mathrm{CAT}(0)$ spaces. The first is the Alexandrov angle. Given two geodesics (segments or rays) $\alpha$ and $\beta$ with the same initial point $p$, the 
Alexandrov angle between them is the angle between their initial velocities (see [4, Definition I.1.12]) and is denoted by $\angle_{p}(\alpha, \beta)$, or $\angle_{p}(a, b)$ if $a$ and $b$ are points on $\alpha$ and $\beta$ other than $p$.

The other notion of an angle is the Tits angle. Given two points $\eta$ and $\zeta$ in the boundary of a CAT( 0$)$ space $Z$ the Tits angle or Tits distance between them is defined by

$$
\angle_{\text {Tits }}(\eta, \zeta)=\sup \left\{\angle_{p}(\overrightarrow{p \eta}, \overrightarrow{p \zeta}) \mid p \in Z\right\}
$$

where $\overrightarrow{p \eta}$ and $\overrightarrow{p \zeta}$ denote the geodesic rays emanating from $p$ going out to $\eta$ and $\zeta$ respectively. In the Euclidean plane the two notions agree: that is, for any point $p$ and geodesic rays $\alpha$ and $\beta$ emanating from $p$ we have:

$$
\angle_{p}(\alpha, \beta)=\angle_{\text {Tits }}(\alpha(\infty), \beta(\infty)) .
$$

In fact, when either angle is less than $\pi$, this equation holds precisely when the convex hull of the union of the two rays is a flat sector [4, Corollary II.9.9].

Finally, the following terminology will be convenient when talking about CAT(0) spaces which split as a product $\Gamma \times \mathbb{R}$ where $\Gamma$ is a tree. For vertices $v \in \Gamma$ we refer to the lines $\{v\} \times \mathbb{R}$ as vertical lines. For a geodesic edge path $v \subset \Gamma$ we refer to the subspace $v \times \mathbb{R}$ as a vertical strip.

\subsection{Knot groups of torus knots}

A torus knot is a knot which lives in a torus. Specifically, given a relatively prime pair $(p, q)$ we let $K=K_{p, q}$ be an imbedding $S^{1} \hookrightarrow T^{2} \subset S^{3}$ which wraps the circle $p$ times around one direction of $T^{2}$ and $q$ times around the other. It follows from the Van Kampen theorem that the fundamental group $G$ of the complement $S^{3}-K$ is presented by:

$$
\left\langle a, b \mid a^{p}=b^{q}\right\rangle .
$$

The center of this group is generated by the element $a^{p}=b^{q}$, which we will denote by $\tau$. Another important group element is the element which represents a meridianal loop in $S^{3}$ around $K$, which we will call $\omega$. By making appropriate choices, we can get

$$
\omega=b^{n} a^{m} \quad \text { where } n, m \text { solve the equation } m q+n p=1 .
$$

As in [4, Example II.11.15(2)], we construct a nonpositively curved $K(G, 1)$. Beginning with a flat rectangle $\bar{R}=[0, \alpha] \times[0, \beta]$ of arbitrary dimensions $\alpha, \beta>0$, we form the 
quotient space $\bar{R} \rightarrow \bar{R} / \sim$ where $\sim$ is generated by the following three relations:

$$
\begin{aligned}
& (0, t) \sim(0, t+\beta / p), \\
& (\alpha, t) \sim(\alpha, t+\beta / q) \text { and } \\
& (t, 0) \sim(t, \beta) .
\end{aligned}
$$

This space is nonpositively curved by [4, Corollary II.11.19]. We denote it by $\bar{Y}$. Note that we get the same result if we use the following construction. Starting with an annulus we glue the two boundary circles to two disjoint circles. One of the attaching maps wraps the circle $p$ times around itself; the other wraps the circle $q$ times around itself. Figure 1 shows these two ways to draw $\bar{Y}$ for the trefoil knot. We observe that $\bar{Y}$ can be realized topologically as a 2-dimensional spine of the complement of $K$.
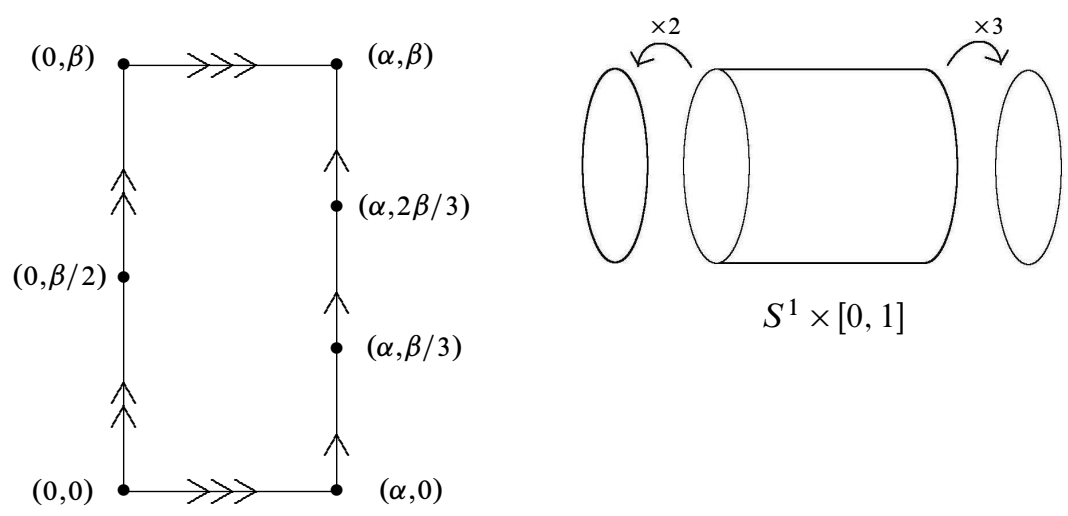

Figure 1: $\bar{Y}$ for the Trefoil Knot

By the Cartan-Hadamard Theorem (proven by S B Alexander and R L Bishop in [1]), the universal cover $p: Y \rightarrow \bar{Y}$ is $\mathrm{CAT}(0)$. This $\mathrm{CAT}(0) G$-space splits the product $\Gamma^{p, q} \times \mathbb{R}$ where $\Gamma^{p, q}$ denotes the $(p, q)$-biregular tree ${ }^{1}$. This is the Bass-Serre tree for the obvious structure as a free product with amalgamation:

$$
G=\langle a\rangle *\langle\tau\rangle\langle b\rangle \text {. }
$$

The action of $G$ on $Y$ is described as follows. The fundamental chamber is a lift $R$ of $\bar{R}$. The isometry $\tau$ is a vertical translation by a distance of $\beta(\operatorname{Min} \tau=Y)$. The axis of $a$ is a vertical line containing one side of $R$. The isometry $a$ is a rotation about this axis followed by a vertical translation by $\beta / p$. Similarly, the isometry $b$ is a rotation about its axis followed by a vertical translation by $\beta / q$. The action of $G$ on $Y$ is

${ }^{1} \mathrm{By}$ “ $(p, q)$-biregular" we mean the infinite tree whose vertices alternate in valence between $p$ and $q$. 
shown in Figure 2. In the picture $v_{a}$ is the fixed point in $\Gamma^{p, q}$ of $a$ and $v_{b}$ is the fixed point in $\Gamma^{p, q}$ of $b$. We choose as our preferred basepoint a point $x_{0}$ in the axis of $a$. Also we coordinatize $Y=\Gamma^{p, q} \times \mathbb{R}$ so that the coordinates of $x_{0}$ are $\left(v_{a}, 0\right)$ and $\tau$ translates in the positive direction of $\mathbb{R}$.

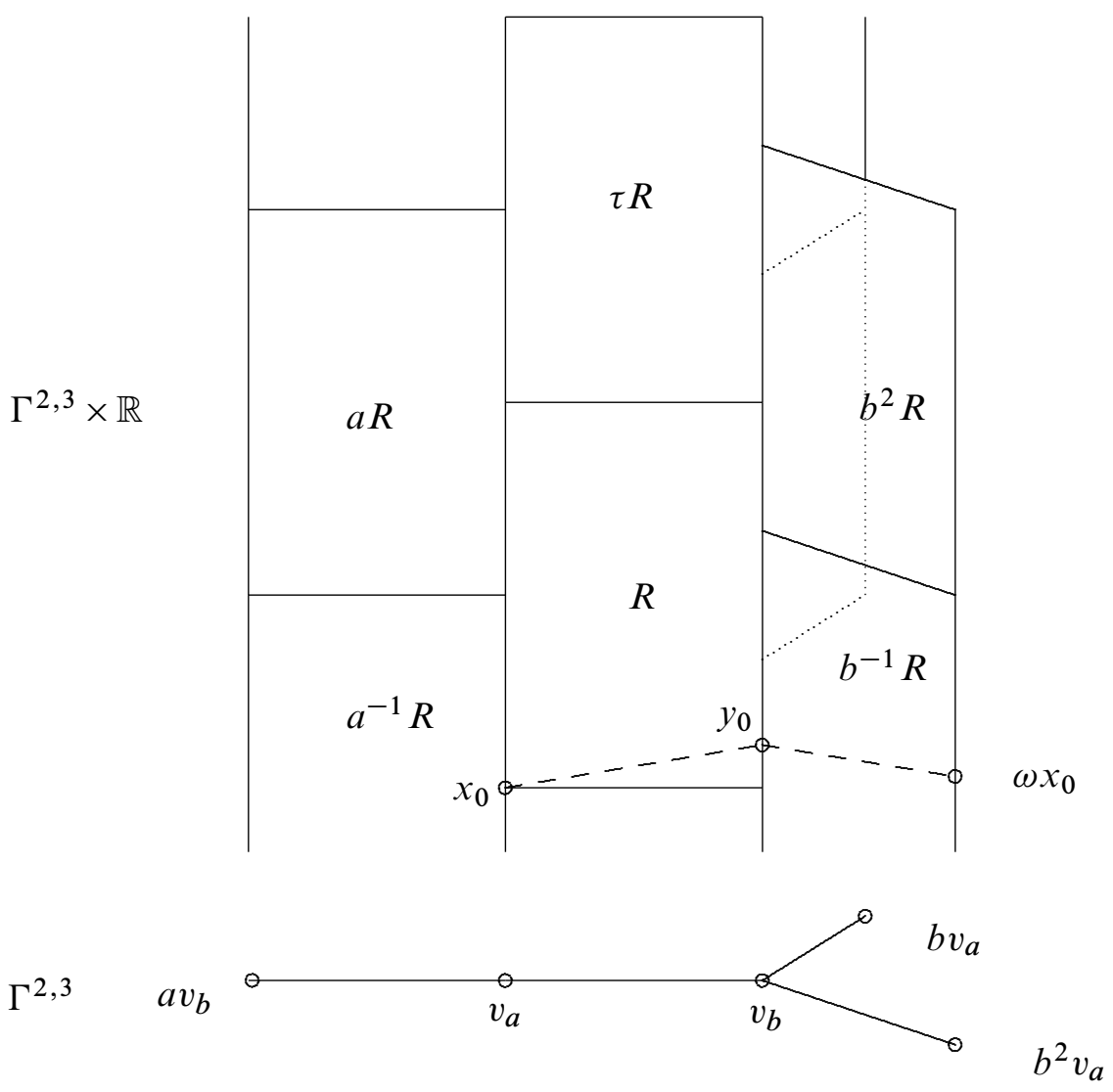

Figure 2: $Y$ in the case $p=2, q=3$

Proposition 4.1 The geodesic $\left[x_{0}, \omega x_{0}\right]$ is the hypotenuse of a right triangle with sides of length $2 \alpha$ and $\beta / p q$. 
Proof Denote the translation vector of a group element $g \in G$ in the $\mathbb{R}$-coordinate by $\lambda(g)$ so that the following hold:

$$
\begin{aligned}
& \lambda(\tau)=\beta, \\
& \lambda(a)=\beta / p \text { and } \\
& \lambda(b)=\beta / q .
\end{aligned}
$$

Recalling that $n$ and $m$ satisfy the equation $m q+n p=1$, we compute:

$$
\begin{aligned}
\lambda(\omega) & =n \lambda(b)+m \lambda(a) \\
& =\frac{n \beta}{q}+\frac{m \beta}{p} \\
& =\frac{\beta}{p q} .
\end{aligned}
$$

So the coordinates of $\omega x_{0}$ in terms of the splitting are:

$$
\omega x_{0}=\left(b^{n} v_{a}, \frac{\beta}{p q}\right) \text {. }
$$

Given $g \in G$ consider the conjugate element $\omega^{g}=g \omega g^{-1}$. Then $\operatorname{Min} \omega^{g}$ is a Euclidean plane of the form $L \times \mathbb{R}$ where $L$ is the (unique) axis of $\omega^{g}$ in $\Gamma^{p, q}$. We call these Euclidean planes walls because they will play the same role as the walls described in Section 2. The geodesic lines $L$ will be called wall shadows.

Lemma 4.1 The number of wall shadows containing a given vertex is equal to the valence of the vertex. The number of wall shadows containing a given edge is 2 .

Proof We begin by proving that the number of wall shadows containing a $p$-valent vertex is $p$. Since $G$ acts transitively on the collection of $p$-valent vertices it suffices to prove this for $v_{a}$. Let $L$ be a wall shadow containing $v_{a}$, say the axis of $\omega^{h}$. Then $L=h L_{0}$ where $L_{0}$ is the axis of $\omega$. Since $h^{-1} v_{a} \in L_{0}$ there is a $k$ such that $\omega^{k} h^{-1} v_{a}=v_{a}$; that is, $\omega^{k} h^{-1}$ fixes $v_{a}$ and is therefore a power of $a$, say $\omega^{k} h^{-1}=a^{-i}$. Then $h=a^{i} \omega^{k}$ and

$$
\omega^{h}=\omega^{a^{i}} .
$$

There are exactly $p$ conjugate elements of this form giving us $p$ wall shadows containing the vertex $v_{a}$. A similar argument works for $q$-valent vertices.

We now prove the second statement of the lemma. Since $G$ acts transitively on the collection of edges, every edge is contained in the same number of wall shadows. Call 
this number $\epsilon$. Consider the number $N$ of pairs $(e, L)$ where $e$ is an edge of the star of $v_{a}$ which is contained in the wall shadow $L$. On one hand since every wall shadow hits two edges of the star of $v_{a}$, we have $N=2 p$. On the other hand since every edge is contained in $\epsilon$ wall shadows, we have $N=\epsilon p$. So $\epsilon=2$.

Lemma 4.2 The intersection of two wall shadows is at most two edges. In fact, two wall shadows can contain more than one edge only when $p$ or $q$ is 2 .

Proof It suffices to prove the following claim.

Claim Let $v$ be any vertex and $L$ and $L^{\prime}$ be two wall shadows containing $v$. Then $L$ and $L^{\prime}$ share two edges of the star of $v$ iff the valence of $v$ is 2 .

By translating the picture we may assume that $v=v_{b}$ or $v_{a}$. Since the argument is the same either way, we will assume $v=v_{b}$. Certainly if $q=2$ then $L$ and $L^{\prime}$ have to share both edges in the star of $v_{b}$. For the converse, assume $L \cap L^{\prime}$ contains two edges in the star of $v_{b}$. Without loss of generality assume one of the edges is $\left[v_{a}, v_{b}\right]$. Then one of the wall shadows is the axis of $\omega$ and the other is the axis of $\omega^{b^{-n}}=a^{m} b^{n}$. Say that $L$ is the former and $L^{\prime}$ is the latter. The two vertices in the link of $v_{b}$ hit by $L$ are $v_{a}$ and $b^{n} v_{a}$. The two vertices in the link of $v_{b}$ hit by $L^{\prime}$ are $v_{a}$ and $b^{-n} v_{a}$. So $L$ and $L^{\prime}$ share two edges in the star of $v_{b}$ only if $b^{n} v_{a}=b^{-n} v_{a}$ which happens precisely when $q \mid 2 n$. Since $q$ and $n$ are relatively prime this is the same as saying that $q=2$. This proves the claim.

Roughly speaking the above lemma tells us that wall shadows bifurcate at odd-valent vertices. Translated into the language of walls this means several things.

Fact 4.1 If $e$ is an edge of $\Gamma^{p, q}$, then the vertical strip $e \times \mathbb{R}$ is contained in exactly two walls.

Fact 4.2 If $v$ is a $p$-valent [ $q$-valent] vertex of $\Gamma^{p, q}$, then the vertical line $v \times \mathbb{R}$ is contained in exactly $p[q]$ walls.

Fact 4.3 Let $W$ and $W^{\prime}$ be walls. Then $W \cap W^{\prime}$ is either empty, a vertical line or a vertical strip.

Let $\gamma_{0}$ denote the axis of $\omega$ containing the point $x_{0}$ and $\bar{\gamma}$ denote its image in $\bar{Y}$. This is a local geodesic loop in $\bar{Y}$ representing $\omega$. The $G$-translates of $\gamma_{0}$ are called joint lines, for reasons which will become apparent in the next section. 


\section{Proposition 4.2 Joint lines do not intersect.}

Proof Suppose two joint lines intersect at a point $z$. Without loss of generality we may assume that one of the joint lines is $\gamma_{0}$ and that $z \in R$. Call the other joint line $\gamma$. Now if $z$ is in the axis of $a$, then $\gamma=a^{k} \gamma_{0}$ for some $k$. But this means that $z=a^{k} z$, which is impossible. A similar argument shows that $z$ cannot be in the axis of $b$. Therefore $z$ is in the open vertical strip $\left(v_{a}, v_{b}\right) \times \mathbb{R}$ and $\gamma=\tau^{k} b^{-n} \gamma_{0}$ for some $k$. If $y_{0}$ is the point at which $\gamma_{0}$ hits the axis of $b$, then

$$
z=\left[x_{0}, y_{0}\right] \cap\left[x_{0}^{\prime}, y_{0}^{\prime}\right],
$$

where $y_{0}^{\prime}=\tau^{k} b^{-n} y_{0}$ and $x_{0}^{\prime}=\tau^{k} a^{m} x_{0}$. Let $r: Y \rightarrow \mathbb{R}$ denote the projection onto the $\mathbb{R}$-coordinate so that $r\left(x_{0}\right)=0$ and $r\left(y_{0}\right)=\beta / 2 p q$. A computation gives $r\left(x_{0}^{\prime}\right)=r\left(y_{0}^{\prime}\right)+\beta / 2 p q$. Therefore:

$$
0<r\left(x_{0}^{\prime}\right) \leq \frac{\beta}{p q} .
$$

But $r\left(x_{0}^{\prime}\right)=i \beta / q$ for some integer $i$, which gives us a contradiction.

Since the axes of a group element are contained in that element's minset, the following is true.

Fact 4.4 Two joint lines are parallel iff they are contained in the same wall.

Here we are using the word "parallel" in the strong Euclidean sense; that is, when we say two lines are parallel we mean that their convex hull is a flat strip. The proof of this next proposition is an immediate consequence of Proposition 4.1.

Proposition 4.3 We can choose the dimensions $\alpha$ and $\beta$ of $\bar{R}$ so that $\bar{\gamma}$ has length 1 and

$$
\angle_{\text {Tits }}\left(\omega^{\infty}, \tau^{\infty}\right)=\angle_{x_{0}}\left(\omega x_{0}, \tau x_{0}\right)=\theta
$$

for any $0<\theta<\pi / 2$ we choose. This done, joint lines form angle $\theta$ with vertical lines.

We close this section with two propositions. The first is recorded to demonstrate the need for Wilson's work [11] as noted at the end of Section 2. The second shows that knot groups of Torus knots are rigid, a fact noted in the introduction.

Proposition 4.4 It is impossible to construct a $C A T(0) G$-space $Y$ in such a way that

$$
\angle_{\text {Tits }}\left(\omega^{\infty}, \tau^{\infty}\right)=\pi / 2
$$


Proof Suppose $Y$ is a CAT(0) $G$-space. Without loss of generality we may assume $Y=\operatorname{Min} \tau$ and splits as $Y=\Gamma^{\prime} \times \mathbb{R}$. Then we can define $\lambda$ as in Proposition 4.1 and compute:

$$
\begin{aligned}
\lambda(\omega) & =n \lambda(b)+m \lambda(a) \\
& =\frac{\lambda(\tau)}{p q} \\
& \neq 0 .
\end{aligned}
$$

Choosing $x_{0} \in \operatorname{Min} \omega$, we have:

$$
\angle_{\text {Tits }}\left(\omega^{\infty}, \tau^{\infty}\right)=\angle_{x_{0}}\left(\omega x_{0}, \tau x_{0}\right)<\frac{\pi}{2} .
$$

\section{Proposition $4.5 G$ is rigid.}

Proof By a result of Bedenikovic, Delgado and Timm [2, Lemma 4.2], we know that $\bar{Y}$ has a nontrivial self-cover. By [2, Theorem 5.2], it has a finite cover $S^{1} \times \mathcal{G} \rightarrow \bar{Y}$ where $\mathcal{G}$ is a finite graph. Therefore $G$ contains the group $F \times \mathbb{Z}$ as a finite index subgroup for some finitely generated free group $F$.

Thus if we are given any $\operatorname{CAT}(0) G$-space $Y$, the induced action of $F \times \mathbb{Z}$ on $Y$ as a subgroup is cocompact and hence geometric. Therefore any $\operatorname{CAT}(0)$ boundary of $G$ is also a boundary of $F \times \mathbb{Z}$. Applying the result of Bowers and Ruane [3], we get that every boundary of $G$ is homeomorphic to the suspension of a cantor set.

\subsection{Knot groups of connected sums of torus knots}

Take two relatively prime pairs $\left(p_{ \pm}, q_{ \pm}\right)$and form the corresponding torus knots $K_{ \pm} \subset S^{3}$. Denote the fundamental group of the complement of $K_{ \pm}$by $G_{ \pm}$and let $\omega_{ \pm} \in G_{ \pm}$denote the group element representing a meridianal loop as in Section 4.2. Let $K$ be a connected sum $K_{-} \# K_{+}$and set

$$
G=\pi_{1}\left(S^{3}-K\right)=G_{-} *_{\mathbb{Z}} G_{+},
$$

where $\mathbb{Z} \hookrightarrow G_{ \pm}$is given by $1 \mapsto \omega_{ \pm}$. Fixing $\theta \in(0, \pi / 2)$, form the $K\left(G_{ \pm}, 1\right)$ prescribed in the previous section and call it $\bar{Y}_{ \pm}$. Construct it so that the local geodesic $\overline{\gamma_{ \pm}} \subset \bar{Y}_{ \pm}$corresponding to the group element $\omega_{ \pm}$has length 1 , and in the universal covers $Y_{ \pm}$of $\bar{Y}_{ \pm}$joint lines form angle $\theta$ with vertical lines. Glue $\bar{Y}_{-}$to $\bar{Y}_{+}$along an isometry $\bar{\gamma}_{-} \cong \bar{\gamma}_{+}$to form a nonpositively curved $K(G, 1)$ which we call $\bar{X}$. Let $p: X \rightarrow \bar{X}$ be the universal covering projection. Then $X$ is a CAT(0) $G$-space. Since $G_{ \pm}$both inject into $G$, the path components of $p^{-1}\left(\bar{Y}_{ \pm}\right)$are isometric copies of $Y_{ \pm}$. 
We call these path components natural blocks. It is easy to see that the collection of natural blocks gives us a block structure on $X$ in the sense of Section 3. Thus the nerve $\mathcal{N}$ of the collection of natural blocks is a tree and we may talk about the itinerary between two natural blocks or the itinerary of a geodesic. We call an itinerary in terms of natural blocks a natural itinerary and use the notation $\operatorname{Itin}_{\mathcal{N}}$.

Now this "natural block structure" is different from the block structure of Croke and Kleiner's construction in [5]. Here natural blocks do not intersect at walls (Euclidean planes) but at joint lines. We will see, however, that the boundary of our construction has the same essential structure as the boundary in [5]. To prove this we will need to introduce another type of block.

Definition 4.1 Given a joint line $\gamma$ we define the joint block of $\gamma$ to be the convex hull of all joint lines $X$ which are parallel to $\gamma$. This done, we define a "new nerve" $\widehat{\mathcal{N}}$ with the following properties.

(1) Vertices $\widehat{v}_{B}$ correspond to blocks $B$ of $X$ (joint and natural).

(2) An edge $\left[\widehat{v}_{B_{1}}, \widehat{v}_{B_{2}}\right]$ is included whenever $B_{1} \cap B_{2}$ is a wall.

When (2) holds we will say that $B_{1}$ neighbors $B_{2}$.

A word of warning: When we call $\widehat{\mathcal{N}}$ a "nerve" we do not mean it in the same sense as used in Section 3. We mean here that it is the correct analogue of the previous notion of a nerve in this context. In [5] at most two blocks could intersect simultaneously and then their intersection was precisely a wall. Here there are many intersections which are not being recorded; for example, every point is in at least three blocks, possibly more. This fact will cause some difficulty for us in Section 5.2 when we need to redefine itineraries of terms of $\widehat{\mathcal{N}}$.

\section{The main theorem}

In order to apply the strategies of Croke and Kleiner [5] and Wilson [11], will need to prove that if we take the collection of all blocks, both joint and natural, together with this "new nerve" $\widehat{\mathcal{N}}$, then Theorem A-Theorem E from Section 2 remain valid. Restated in this context the theorems will be labeled Theorem $\mathrm{A}^{\prime}-$ Theorem $\mathrm{E}^{\prime}$. 


\subsection{Joint blocks}

If $\left[B_{1}, \ldots, B_{n}\right]$ is a natural itinerary, then we call the list of joint lines

$$
\begin{gathered}
\gamma_{1}=B_{1} \cap B_{2} \\
\gamma_{2}=B_{2} \cap B_{3} \\
\vdots \\
\gamma_{n-1}=B_{n-1} \cap B_{n}
\end{gathered}
$$

the list of joint lines between $B_{1}$ and $B_{n}$. If $\gamma$ and $\gamma^{\prime}$ are two joint lines, then it is easy to see that every geodesic which begins on $\gamma$ and ends on $\gamma^{\prime}$ has the same itinerary. If that itinerary is $\left[B_{1}, \ldots, B_{n}\right]$, and $\gamma_{1}, \ldots, \gamma_{n-1}$ is the list of joint lines between $B_{1}$ and $B_{n}$, then we also call $\gamma_{1}, \ldots, \gamma_{n-1}$ the the list of joint lines between $\gamma$ and $\gamma^{\prime}$.

Lemma 5.1 (The joint line lemma) Let $\gamma$ and $\gamma^{\prime}$ be parallel joint lines. Then every joint line between $\gamma$ and $\gamma^{\prime}$ is also parallel to $\gamma$ and $\gamma^{\prime}$.

Proof Parameterize $\gamma, \gamma^{\prime}: \mathbb{R} \rightarrow X$ to have unit speed and let $\gamma_{0}$ be a joint line between $\gamma$ and $\gamma^{\prime}$. Then every geodesic which begins on $\gamma$ and ends on $\gamma^{\prime}$ must pass through $\gamma_{0}$ (Lemma 3.2(2)). In particular, for $k \in \mathbb{Z}$ the geodesic $\left[\gamma(k), \gamma^{\prime}(k)\right]$ intersects $\gamma_{0}$ at some point $z_{k}$. Since $\gamma \| \gamma^{\prime}, d\left(\gamma(k), \gamma^{\prime}(k)\right)$ is constant and $z_{k}$ remains asymptotic to $\gamma$ and $\gamma^{\prime}$ as $k \rightarrow \pm \infty$. It follows that $\gamma_{0}$ is indeed parallel to these.

Proposition 5.1 Let $B_{J}$ be the joint block of a joint line $\gamma_{0}$. Then the following hold.

(1) $B_{J} \cong \Gamma^{4} \times \mathbb{R}$ where $\Gamma^{4}$ is the 4-valent tree.

(2) The joint lines parallel to $\gamma_{0}$ are precisely the vertical lines in $B_{J}$.

(3) If a joint line $\gamma \subset B_{J}$, then $\gamma \| \gamma_{0}$.

Proof Let $D^{0}=\gamma_{0}$, and for each $n>0$, let $D^{n}$ denote the union of $D^{n-1}$ along with all walls intersecting $D^{n-1}$ at a joint line. In addition, we define $D^{\infty}=\bigcup_{i=0}^{\infty} D^{i}$. Since two nonintersecting lines in a common Euclidean plane must be parallel, we see that $D^{n}$ splits as $T^{n} \times \mathbb{R}$ where each $T^{n}$ is a tree, constructed as follows: We begin with $T^{0}$, which is just a point. $T^{1}$ is the union of two lines glued together at a single point $z_{0}$. To form $T^{2}$, we glue four new lines to $T^{1}$ at four points $z_{1}, \ldots, z_{4}$ in the four components of $T^{1}-z_{0}$. To form $T^{3}$, we glue twelve new lines to $T^{2}$ at twelve points in the twelve unbounded components of $T^{2}-\left\{z_{1}, \ldots, z_{4}\right\}$, and so on. The limit $\Gamma^{4}$ of this increasing sequence of trees is an infinite 4-valent tree. Thus we get $D^{\infty}=\Gamma^{4} \times \mathbb{R}$. Furthermore, we see from the construction that the joint lines 
in $D^{\infty}$ are precisely the vertical lines, and that all of these are parallel to $\gamma_{0}$. So the proposition will follow if we show that $D^{\infty}=B_{J}$.

Certainly $D^{\infty}$ is the convex hull of the collection of joint lines parallel to $\gamma_{0}$ which are contained in $D^{\infty}$. What we need to know is that all joint lines parallel to $\gamma_{0}$ are contained in $D^{\infty}$. We prove this here: Let $\gamma$ be a joint line parallel to $\gamma_{0}$, and $\gamma_{1}, \ldots, \gamma_{n-1}$ be the list of joint lines between $\gamma_{0}$ and $\gamma$. Since $\gamma_{1} \| \gamma_{0}$ and these two are in a common natural block, it follows that they are in a common wall and that $\gamma_{1} \subset D^{1}$. In general, since $\gamma_{i} \| \gamma_{i+1}$ and these two joint lines are in a common natural block, they are in a common wall and therefore $\gamma_{i+1} \subset D^{i+1}$. So $\gamma_{n-1} \subset D^{n-1}$ and $\gamma \subset D^{n}$.

Remark 5.1 This proposition corresponds to the group theoretic fact that the stabilizer of a joint block is $[\mathbb{Z} * \mathbb{Z}] \times \mathbb{Z}$. For example, if $\gamma_{0}$ is the joint line containing $x_{0}$ then the stabilizer of $B_{J}$ is $\left[\left\langle\tau_{-}\right\rangle *\left\langle\tau_{+}\right\rangle\right] \times\langle\omega\rangle$ where $\omega$ translates $B_{J}$ in the $\mathbb{R}$-direction and $\left\langle\tau_{-}\right\rangle *\left\langle\tau_{+}\right\rangle$acts geometrically on $\Gamma^{4}$. Here $\left\langle\tau_{ \pm}\right\rangle$denote the centers of $G_{ \pm}$.

Two distinct blocks neighbor each other iff one is joint, the other is natural, and the two share a joint line. For a joint block $B_{J}$ let $\mathcal{C}\left(B_{J}\right)$ denote the collection of natural blocks which neighbor $B_{J}$ and $\mathcal{N}\left(B_{J}\right)$ denote the full subgraph of $\mathcal{N}$ spanned by the vertices $\left\{v_{B_{N}} \mid B_{N} \in \mathcal{C}\left(B_{J}\right)\right\}$.

Lemma 5.2 Let $B_{J}$ and $B_{J}^{\prime}$ be distinct joint blocks. The following hold.

(1) If $\left[v_{B_{N}}, v_{B_{N}^{\prime}}\right]$ is an edge of $\mathcal{N}\left(B_{J}\right)$, then the joint line $B_{N} \cap B_{N}^{\prime}$ is in $B_{J}$.

(2) If $B_{N}, B_{N}^{\prime} \in \mathcal{C}\left(B_{J}\right)$, then $\operatorname{Itin}_{\mathcal{N}}\left[B_{N}, B_{N}^{\prime}\right] \subset \mathcal{C}\left(B_{J}\right)$.

(3) $\left|\mathcal{C}\left(B_{J}\right) \cap \mathcal{C}\left(B_{J}^{\prime}\right)\right| \leq 1$.

\section{Proof}

(1) Let $\gamma$ and $\gamma^{\prime}$ be joint lines of $B_{N}$ and $B_{N}^{\prime}$ which are in $B_{J}$. Since $B_{N} \cap B_{N}^{\prime}=$ $\gamma_{0}$ is a joint line, $\gamma_{0}$ is the only joint line between $\gamma$ and $\gamma^{\prime}$. It follows from the joint line lemma that $\gamma_{0}$ is parallel to $\gamma$ and $\gamma^{\prime}$ and must therefore also be in $B_{J}$.

(2) Again, let $\gamma$ and $\gamma^{\prime}$ be joint lines of $B_{N}$ and $B_{N}^{\prime}$ which are in $B_{J}$, and write $\operatorname{Itin}_{\mathcal{N}}\left[B_{N}, B_{N}^{\prime}\right]=\left[B_{1}, \ldots, B_{k}\right]$. Then for $1 \leq i<k$, the joint line lemma tells us that the joint lines $B_{i} \cap B_{i+1}$ are all in $B_{J}$. So for $1 \leq i \leq k$, every $B_{i}$ shares a joint line with $B_{J}$.

(3) Suppose $\left|\mathcal{C}\left(B_{J}\right) \cap \mathcal{C}\left(B_{J}^{\prime}\right)\right|>1$. Since $\mathcal{N}\left(B_{J}\right)$ and $\mathcal{N}\left(B_{J}^{\prime}\right)$ are convex, $\mathcal{N}\left(B_{J}\right) \cap \mathcal{N}\left(B_{J}^{\prime}\right)$ must contain an edge $\left[v_{B_{N}}, v_{B_{N}^{\prime}}\right]$; by (1), the joint line $B_{N} \cap B_{N}^{\prime}$ is in both $B_{J}$ and $B_{J}^{\prime}$, which is a contradiction. 


\subsection{Itineraries in $\widehat{\mathcal{N}}$}

Our goal here is to show that $\widehat{\mathcal{N}}$ is a tree (Theorem $\mathrm{A}^{\prime}$ ) and to define itineraries in terms of $\widehat{\mathcal{N}}$.

Lemma 5.3 Let $\left[\widehat{v}_{B_{1}}, \ldots, \widehat{v}_{B_{n}}\right]$ be an edge path in $\widehat{\mathcal{N}}$ with no backtracking such that $B_{1}$ and $B_{n}$ are natural blocks. Then

$$
\begin{aligned}
\operatorname{Itin}_{\mathcal{N}}\left[B_{1}, B_{n}\right] & =\operatorname{Itin}_{\mathcal{N}}\left[B_{1}, B_{3}\right] \cup \operatorname{Itin}_{\mathcal{N}}\left[B_{3}, B_{5}\right] \cup \\
& \cdots \cup \operatorname{Itin}_{\mathcal{N}}\left[B_{n-4}, B_{n-2}\right] \cup \operatorname{Itin}_{\mathcal{N}}\left[B_{n-2}, B_{n}\right],
\end{aligned}
$$

where

$$
\operatorname{Itin}_{\mathcal{N}}\left[B_{i-1}, B_{i+1}\right] \subset \mathcal{C}\left(B_{i}\right)
$$

for even $1<i<n$.

Proof We prove this by induction on $n$. When $n=1$, there is nothing to show, and when $n=3$, we simply note that $\mathcal{N}\left(B_{2}\right)$ is convex. Assume $n \geq 5$, and let $\eta$ denote the geodesic edge path in $\mathcal{N}$ from $v_{B_{1}}$ to $v_{B_{n-2}}$; since $B_{n-4} \neq B_{n-2}$, the last edge of $\eta$ is in $\mathcal{N}\left(B_{n-3}\right)$ (by induction). It follows that $\eta \cap \mathcal{N}\left(B_{n-1}\right)=\left\{v_{B_{n-2}}\right\}$. Hence, if $\eta^{\prime}$ is the geodesic edge path from $v_{B_{n-2}}$ to $v_{B_{n}}$, then since $\eta^{\prime} \subset \mathcal{N}\left(B_{n-1}\right)$, the edge path $\eta \cup \eta^{\prime}$ has no backtracking and must be the geodesic edge path in $\mathcal{N}$ between $v_{B_{1}}$ and $v_{B_{n}}$.

Theorem $\mathbf{A}^{\prime} \widehat{\mathcal{N}}$ is a tree.

Proof It follows from Lemma 5.2(3) that $\widehat{N}$ has no squares. Thus, any non-nullhomotopic loop in $\widehat{N}$ must have length at least 6 . Suppose $\left[\widehat{v}_{B_{1}}, \ldots, \widehat{v}_{B_{n}}\right]$ is such a loop with no backtracking where $B_{1}=B_{n}$ is natural. Then by the previous lemma, the first edge in the geodesic edge path in $\mathcal{N}$ from $v_{B_{1}}$ to $v_{B_{3}}$ is in both $\mathcal{N}\left(B_{2}\right)$ and $\mathcal{N}\left(B_{n-1}\right)$, giving us a contradiction.

Proposition 5.2 Suppose $\left[\widehat{v}_{B_{1}}, \ldots, \widehat{v}_{B_{n}}\right]$ is a geodesic edge path in $\widehat{\mathcal{N}}$ and $\alpha$ is a geodesic segment which begins in $B_{1}$ and ends in $B_{n}$. Then then $\alpha$ is covered by the collection of blocks $\left\{B_{k}\right\}_{k=1}^{n}$ and passes through every block $B_{k}$ and wall $B_{k} \cap B_{k+1}$.

Proof First of all, assume $B_{1}$ and $B_{n}$ are both natural blocks and use Lemma 5.3 to write:

$$
\begin{aligned}
\operatorname{Itin}_{\mathcal{N}} \alpha & \subset \operatorname{Itin}_{\mathcal{N}}\left[B_{1}, B_{n}\right] \\
& =\operatorname{Itin}_{\mathcal{N}}\left[B_{1}, B_{3}\right] \cup \cdots \cup \operatorname{Itin}_{\mathcal{N}}\left[B_{n-2}, B_{n}\right] .
\end{aligned}
$$


So for every odd $1<k<n, \alpha$ passes through the block $B_{k}$. Let $\gamma_{k-2}$ denote the joint line at which $\alpha$ leaves the natural block $B_{k-2}$ and $\gamma_{k-1}$ denote the joint line at which $\alpha$ enters the natural block $B_{k}$. The fact that $\operatorname{Itin}_{\mathcal{N}}\left[B_{k-2}, B_{k}\right] \subset C\left(B_{k-1}\right)$ tells us that $\gamma_{k-2}, \gamma_{k-1} \subset B_{k-1}$. For every $1 \leq k<n$, let $t_{k}$ be the time such that $\alpha\left(t_{k}\right) \in \gamma_{k}$. Since $\gamma_{k} \subset B_{k} \cap B_{k+1}$, we see that $\alpha$ hits every such wall. Furthermore,

$$
\gamma\left(\left[t_{k}, t_{k+1}\right]\right) \subset B_{k},
$$

because $B_{k}$ is convex. This shows that $\alpha \subset \bigcup_{k=1}^{n} B_{k}$.

Now consider the more general case. If $B_{1}$ is joint and $B_{n}$ is natural, choose a natural block $B_{0}$ containing the initial point of $\alpha$. This time Lemma 5.3 gives us:

$$
\begin{aligned}
\operatorname{Itin}_{\mathcal{N}} \alpha & \subset \operatorname{Itin}_{\mathcal{N}}\left[B_{0}, B_{n}\right] \\
& =\operatorname{Itin}_{\mathcal{N}}\left[B_{0}, B_{2}\right] \cup \operatorname{Itin}_{\mathcal{N}}\left[B_{2}, B_{4}\right] \cdots \cup \operatorname{Itin}_{\mathcal{N}}\left[B_{n-2}, B_{n}\right] .
\end{aligned}
$$

As before, since $\alpha$ enters $B_{2}$ at a joint line of $B_{1}$, we get that $\alpha$ passes through the wall $B_{1} \cap B_{2}$ and because blocks are convex, we get that $\alpha \subset \bigcup_{k=1}^{n} B_{k}$. Similar arguments work if $B_{1}$ is natural and $B_{n}$ is joint, or if both $B_{1}$ and $B_{n}$ are joint.

We now know that given a geodesic segment (or ray) $\alpha$ there is a (possibly infinite) geodesic edge path $\left[\widehat{v}_{B_{1}}, \ldots, \widehat{v}_{B_{n}}\right]$ such that $\alpha \subset \bigcup_{k=1}^{n} B_{k}$. We define the $\widehat{\mathcal{N}}-$ itinerary of $\alpha$ to be the list $\left[B_{1}, \ldots, B_{n}\right]$ where $\left[\widehat{v}_{B_{1}}, \ldots, \widehat{v}_{B_{n}}\right]$ is the shortest such

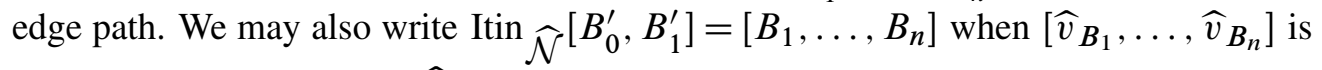
the geodesic edge path in $\widehat{\mathcal{N}}$ from $\widehat{v}_{B_{0}^{\prime}}$ to $\widehat{v}_{B_{1}^{\prime}}$.

There is some danger of confusion here since every geodesic in $X$ has two itineraries: one in terms of $\mathcal{N}$ and the other in terms of $\widehat{\mathcal{N}}$. We already have a notion of rational and irrational rays in terms of $\mathcal{N}$-itineraries. We denote the set of points which are "rational with respect to $\mathcal{N}$ " by $R_{\mathcal{N}} X$ and the set of points which are "irrational with respect to $\mathcal{N}$ " by $I_{\mathcal{N}} X$. However, in this section we will call a geodesic ray rational if its $\widehat{\mathcal{N}}$-itinerary is finite and irrational if its $\widehat{\mathcal{N}}$-itinerary is infinite. The endpoint of a rational ray is called a rational point and the endpoint of an irrational ray is called an irrational point. To emphasize that by "rational" and "irrational" we mean in terms of $\widehat{\mathcal{N}}$, we will denote the set of rational points of $\partial X$ by $R \widehat{\mathcal{N}}^{X \text { and the set of irrational }}$ points of $\partial X$ by $I \widehat{\mathcal{N}}^{X}$.

Proposition 5.3 If $\alpha$ and $\beta$ are two irrational geodesic rays whose $\widehat{\mathcal{N}}$-itineraries eventually coincide, then their $\mathcal{N}$-itineraries are also infinite and also eventually coincide. 
Proof Suppose we have two irrational geodesic rays $\alpha$ and $\beta$ whose itineraries eventually coincide. Write

$$
\operatorname{Itin}_{\widehat{\mathcal{N}}} \alpha=\left[B_{1}, B_{2}, \ldots\right]
$$

and:

$$
\operatorname{Itin}_{\widehat{\mathcal{N}}} \beta=\left[B_{1}^{\prime}, B_{2}^{\prime}, \ldots\right] .
$$

Then there are $m, n>2$ such that $B_{m+i}=B_{n+i}^{\prime}$ for $i \geq 0$. Choose $m$ and $n$ so that $B_{m}=B_{n}^{\prime}$ is a natural block. The fact that $m, n>2$ guarantees that $\alpha$ and $\beta$ do not begin in this block; hence $B_{m} \in \operatorname{Itin}_{\mathcal{N}} \alpha \cap \operatorname{Itin}_{\mathcal{N}} \beta$. In fact, $B_{m+2 i} \in \operatorname{Itin}_{\mathcal{N}} \alpha \cap \operatorname{Itin}_{\mathcal{N}} \beta$ for every $i \geq 0$. Therefore $\operatorname{Itin}_{\mathcal{N}} \alpha \cap \operatorname{Itin}_{\mathcal{N}} \beta$ contains the infinite sequence of blocks $\left\{B_{m}, B_{m+1}, B_{m+2}, \ldots\right\}$.

\section{Corollary $5.1 \quad$ (1) $I_{\widehat{\mathcal{N}}} X \subset I_{\mathcal{N}} X$.}

(2) $R_{\mathcal{N}} X \subset R_{\widehat{\mathcal{N}}} X$.

Remark 5.2 The above inclusions are strict. A geodesic ray which stays in the same joint block but does not stay in any wall will have an infinite $\mathcal{N}$-itinerary but finite $\widehat{\mathcal{N}}$-itinerary.

\subsection{The boundary of $X$}

We do not yet know that $R \widehat{\mathcal{N}}^{X}$ is precisely the union of block boundaries. For this we need to know Lemma 3.3 in the new context. The proof is the same except that we replace the claim with the following lemma.

Lemma 5.4 There is a $\delta>0$ such that for natural blocks $B$ and $B^{\prime}$ if $d_{\widehat{\mathcal{N}}}\left(\widehat{v}_{B}, \widehat{v}_{B^{\prime}}\right) \geq$ $4 k$ then $d\left(B, B^{\prime}\right) \geq k \delta$.

Proof Let $\delta$ be the minimum positive distance between joint lines in $X$. Let $B$ and $B^{\prime}$ be natural blocks, $\operatorname{Itin}_{\mathcal{N}}\left[B, B^{\prime}\right]=\left[B_{0}, \ldots, B_{n}\right]$ where $n \geq 4 k, x \in B$ and $x^{\prime} \in B^{\prime}$. Then the geodesic $\left[x, x^{\prime}\right]$ passes through every block $B_{4 i}$ for $0 \leq i \leq k$ at some point $z_{i}$. Furthermore, for $0 \leq i<k$ the geodesic $\left[z_{i}, z_{i+1}\right]$ enters the block $B_{4 i+2}$ at a joint line of the joint block $B_{4 i+1}$ and leaves at a joint line of the block $B_{4 i+3}$. Thus we have

$$
d\left(x, x^{\prime}\right) \geq \sum_{i=0}^{k-1} d\left(z_{i}, z_{i+1}\right) \geq k \delta .
$$

Corollary 5.2 $R_{\widehat{\mathcal{N}}}^{X}$ is the union of block boundaries and $I \widehat{\mathcal{N}}^{X}$ is its complement. 
Since every block $B$ splits as $\Gamma \times \mathbb{R}$ for some tree $\Gamma$ (one of $\Gamma^{4}, \Gamma^{p_{-}, q_{-}}$or $\Gamma^{p_{+}, q_{+}}$), it follows that $\partial B$ is the suspension of a cantor set. As mentioned in Section 2, the suspension points are called poles and the set of poles is denoted $P B$.

Theorem $\mathbf{B}^{\prime} \quad$ Let $B_{0}$ and $B_{1}$ be blocks and $D$ be the distance between the corresponding vertices in $\widehat{N}$.

(1) If $D=1$, then $\partial B_{0} \cap \partial B_{1}=\partial W$ where $W$ is the wall $B_{0} \cap B_{1}$.

(2) If $D=2$, then $\partial B_{0} \cap \partial B_{1}=P B_{1 / 2}$ where $B_{1 / 2}$ neighbors both $B_{0}$ and $B_{1}$.

(3) If $D>2$, then $\partial B_{0} \cap \partial B_{1}=\varnothing$.

Proof (1) If $D=1$, then $B_{0} \cap B_{1}$ is a wall $W$. That $\partial W \subset \partial B_{0} \cap \partial B_{1}$ is obvious. The reverse inclusion follows by the same sort of argument as was used in the joint line lemma: If $\alpha_{0} \subset B_{0}$ and $\alpha_{1} \subset B_{1}$ are asymptotic geodesic rays, then every geodesic from $\alpha_{0}$ to $\alpha_{1}$ intersects the wall $W$. Thus we can get a sequence of points in $W$ which remain asymptotic to $\alpha_{0}$ and $\alpha_{1}$.

(2) If $D=2$, then there is one vertex between $\widehat{v}_{B_{0}}$ and $\widehat{v}_{B_{1}}$; call it $\widehat{v}_{B_{1 / 2}}$. We will show that

$$
P B_{1 / 2} \subset \partial B_{0} \cap \partial B_{1} \subset \partial W_{0} \cap \partial W_{1} \subset P B_{1 / 2},
$$

where $W_{i}=B_{1 / 2} \cap B_{i}$ for $i=0,1$. The first inclusion is just the fact that vertical lines of $B_{1 / 2}$ can be found in $W_{0}$ and $W_{1}$, and the second is the same argument as in (1). For the third inclusion, suppose $\alpha_{0} \subset W_{0}$ and $\alpha_{1} \subset W_{1}$ are asymptotic geodesic rays and let $\bar{\alpha}_{0}$ and $\bar{\alpha}_{1}$ be the projections of $\alpha_{0}$ and $\alpha_{1}$ onto the $\Gamma$-coordinate of $B_{1 / 2}=\Gamma \times \mathbb{R}$. If $\bar{\alpha}_{0}$ and $\bar{\alpha}_{1}$ are not constant, then since they are asymptotic they must have infinitely many vertices of $\Gamma$ in common. In this case $W_{0} \cap W_{1}$ shares a half-plane, contradicting the fact that $W_{0} \cap W_{1}$ is at most a vertical strip. So $\bar{\alpha}_{0}$ and $\bar{\alpha}_{1}$ are constant and $\alpha_{0}$ and $\alpha_{1}$ go to a pole of $B_{1 / 2}$.

Finally we show (3) by contradiction: Suppose $\zeta \in \partial B_{0} \cap \partial B_{1}$ and write

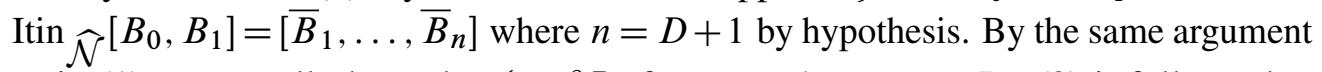
as in (1) we actually have that $\zeta \in \partial B_{i}$ for every $1 \leq i \leq n$. By (2) it follows that $\zeta \in P B_{i}$ for every $1<i<n$. But $P B_{2} \cap P B_{3}=\varnothing$ because $\angle_{\text {Tits }}\left(P B_{2}, P B_{3}\right)=\theta$, giving us a contradiction!

Theorem $C^{\prime} \quad$ Let $B$ be a block and $\zeta \in \partial B$ not be a pole of any neighboring block. Then $\zeta$ has a local path component which stays in $\partial B$.

Proof The proof is the same as the proof of Theorem $\mathrm{C}$ with one minor exception. It could be that a geodesic ray may exit a wall $W$ via a joint line $\gamma$ of another wall. But by Fact $4.3, W \cap \gamma$ is compact in this case. So this exception causes no problems. 
As in [5] we call $\zeta \in \partial X$ a vertex if there is a local path component $V$ of $\zeta$ and a local path component $V^{\prime}$ of an actual pole $\zeta^{\prime}$ and a homeomorphism $(V, \zeta) \approx\left(V^{\prime}, \zeta^{\prime}\right)$. A point of $\partial X$ is a vertex iff it has a local path component homeomorphic to the open cone on the cantor set via a homeomorphism which takes $\zeta$ to the cone point. A path in $\partial X$ is called safe if it passes through vertices at only finitely many times. Since $R \widehat{\mathcal{N}}^{X}$ is just the union of block boundaries (Corollary 5.2), Theorem $\mathrm{C}^{\prime}$ tells us that the only vertices in $R_{\widehat{\mathcal{N}}} X$ are poles.

Theorem $\mathbf{D}^{\prime} \quad R \widehat{\mathcal{N}}^{X}$ is the unique dense safe path component of $\partial X$.

Proof The proof that $R_{\widehat{\mathcal{N}}} X$ is a safe path component is exactly the same as the proof of [5, Lemma 6]. The fact that $R_{\widehat{\mathcal{N}}} X$ is dense follows from Lemma 3.4 and the fact that $R_{\widehat{\mathcal{N}}} X \supset R_{\mathcal{N}} X$. Now the other safe path components are contained in the path components of $I \widehat{\mathcal{N}}^{X}$. Recall that Corollary 3.2 provided us with a map $\phi: I_{\mathcal{N}} X \rightarrow \partial \mathcal{N}$ which is "irrational with respect to $\mathcal{N}$ ". By Proposition 5.2 we know that the restriction $\widehat{\phi}$ of $\phi$ to $I \widehat{\mathcal{N}}^{X}$ is "irrational with respect to $\widehat{\mathcal{N}}$ ". Since $\widehat{\phi}$ takes safe path components to points and no point of im $\widehat{\phi}$ is dense in im $\widehat{\phi}$ it follows that no safe path component of $I \widehat{\mathcal{N}}^{X}$ is dense in $I \widehat{\mathcal{N}}^{X}$.

\section{Theorem $\mathbf{E}^{\prime} \quad$ Let $B$ be a block.}

(1) The union of boundaries of walls of $B$ is dense in $\partial B$.

(2) The closure of the set of poles of neighboring blocks is the same as the set of points of $\partial B$ which are a Tits distance of $\theta$ from a pole of $B$.

Proof Let $\alpha$ be any geodesic ray in $B=\Gamma \times \mathbb{R}$ and $\bar{\alpha}$ be its projection onto the $\Gamma$ coordinate. Let $\bar{\alpha}\left(t_{1}\right), \bar{\alpha}\left(t_{2}\right), \ldots$ be the sequence of non-bivalent vertices through which $\bar{\alpha}$ passes. Then for every $n \geq 1$ there is a wall $W_{n}$ such that $\alpha\left(\left[0, t_{n}\right]\right) \cap W_{n}=\alpha\left(t_{n}\right)$. Thus we may bifurcate $\alpha$ at $\alpha\left(t_{n}\right)$ to get a (probably new) ray $\alpha_{n}$ which agrees with $\alpha$ up to time $t_{n}$ and then stays in $W_{n}$. This proves (1).

For (2) assume that in the above setup we have $\angle_{\text {Tits }}(\alpha(\infty), \zeta)=\theta$ where $\zeta$ is a pole of $B$. This means that $\alpha$ hits vertical lines of $B$ at an angle of $\theta$. Since $\alpha$ enters the wall $W_{n}$ at time $t_{n}$ we have two choices for $\alpha_{n}$. For one of these choices we will have $\alpha_{n}\left(\left[t_{n}, \infty\right)\right)$ parallel to the non-vertical lines in $W_{n}$. Then $\alpha_{n}(\infty)$ will be a pole of a neighboring block.

Theorem 1 The knot group $G$ of any connected sum of two non-trivial torus knots has uncountably many $C A T(0)$ boundaries. 
Proof We sketch here the key argument of [11]. For $0<\theta<\pi / 2$ construct $X_{\theta}$ as above. Now suppose we have $0<\theta_{1}, \theta_{2}<\pi / 2$ and a homeomorphism $h: \partial X_{\theta_{1}} \rightarrow \partial X_{\theta_{2}}$. Since $h$ takes vertices to vertices it follows from Theorem D' that it takes $R \widehat{\mathcal{N}}^{X_{\theta_{1}}}$ to $R \widehat{\mathcal{N}}_{\theta_{2}}$. From here it is not hard to see that $h$ takes poles to poles, block boundaries to block boundaries and wall boundaries to wall boundaries. Let $W_{1}$ be a wall in $X_{\theta_{1}}$ and $W_{2}$ be the wall of $X_{\theta_{2}}$ such that $h\left(\partial W_{1}\right)=\partial W_{2}$. Using Theorem $\mathrm{E}^{\prime}$ and a proof by induction we find sequence of points $\left(z_{k}\right)_{k=0}^{\infty} \subset \partial W_{1}$ such that

$$
\begin{aligned}
\angle_{\text {Tits }}\left(z_{k}, z_{k+1}\right) & =\theta_{1} \\
\text { and } \quad \angle_{\text {Tits }}\left(h\left(z_{k}\right), h\left(z_{k+1}\right)\right) & =\theta_{2} .
\end{aligned}
$$

If $\theta_{1}$ is a rational multiple of $\pi$, then $\left\{z_{k}\right\}$ is a finite set and we can use a counting argument to prove that $\theta_{1}=\theta_{2}$. If $\theta_{1}$ is not a rational multiple of $\pi$, then $\left\{z_{k}\right\}$ is a dense subset of $\partial W_{1}$ and the same argument no longer works. Wilson's solution is to use the sequences $\left(z_{k}\right)$ and $\left(h\left(z_{k}\right)\right)$ to define two nonstandard orderings of the natural numbers denoted $\prec_{1}$ and $\prec_{2}$ such that $\prec_{1}$ is equivalent to $\prec_{2}$. She then uses a technical argument to show that this fact implies that $\theta_{1}=\theta_{2}$.

\section{References}

[1] S B Alexander, R L Bishop, The Hadamard-Cartan theorem in locally convex metric spaces, Enseign. Math. (2) 36 (1990) 309-320 MR1096422

[2] T Bedenikovic, A Delgado, M Timm, A classification of 2-complexes with nontrivial self-covers, Topology Appl. 153 (2006) 2073-2091 MR2239071

[3] P L Bowers, K Ruane, Boundaries of nonpositively curved groups of the form $G \times \mathbf{Z}^{n}$, Glasgow Math. J. 38 (1996) 177-189 MR1397173

[4] M R Bridson, A Haefliger, Metric spaces of non-positive curvature, Grundlehren series 319, Springer, Berlin (1999) MR1744486

[5] C B Croke, B Kleiner, Spaces with nonpositive curvature and their ideal boundaries, Topology 39 (2000) 549-556 MR1746908

[6] C B Croke, B Kleiner, The geodesic flow of a nonpositively curved graph manifold, Geom. Funct. Anal. 12 (2002) 479-545 MR1924370

[7] T Hosaka, On splitting theorems for CAT(O) spaces and compact geodesic spaces of non-positive curvature arXiv:math/0405551

[8] G C Hruska, Geometric invariants of spaces with isolated flats, Topology 44 (2005) 441-458 MR2114956

[9] K E Ruane, Boundaries of CAT(0) groups of the form $\Gamma=G \times H$, Topology Appl. 92 (1999) 131-151 MR1669823 
[10] W P Thurston, Three-dimensional manifolds, Kleinian groups and hyperbolic geometry, Bull. Amer. Math. Soc. (N.S.) 6 (1982) 357-381 MR648524

[11] J M Wilson, A CAT(0) group with uncountably many distinct boundaries, J. Group Theory 8 (2005) 229-238 MR2126732

Department of Mathematical Sciences, University of Wisconsin-Milwaukee PO Box 413, Milwaukee, Wisconsin 53201-0413, USA

cpmooney@uwm.edu

Received: 14 June 2007 Revised: 30 May 2008 